\title{
Sensorless Torque Estimation in Multidegree-of-Freedom Flexible Systems
}

\author{
Islam S. M. Khalil, E. D. Kunt, Asif Sabanovic \\ Faculty of Engineering and Natural Science-Mechatronics Engineering \\ Sabanci University \\ Orhanli-Tuzla, 34956, Istanbul, Turkey \\ Email: kahalil,edkunt@su.sabanciuniv.edu, asif@sabanciuniv.edu
}

\begin{abstract}
This paper presents a sensorless torque estimation algorithm for multidegree-of-freedom œexible systems. The proposed algorithm makes it possible to estimate externally applied torques due to axible system $s$ interaction with the environment without taking any measurement from the system. The algorithm is based on modifying the disturbance observer in order to decouple the reoected torque waves out of the total disturbance on the actuator. Then Reoected torque waves are used along with the actuator $s$ current and velocity to estimate oexible system parameters, dynamics and the external torques or disturbances. Several experimental results are included in order to con $6 \mathrm{rm}$ the validity of the proposed torque estimation algorithm.
\end{abstract}

\section{INTRODUCTION}

Torque and force control have been used in wide variety of applications such as machining tasks, assembly and grasping. And typically these applications requires using a force sensor to provide the controller with a force feed back signal. However, force sensor has certain disadvantages and prevent realization of precise force control. Especially, sensor noise that causes degradation of control performance [1]. Therefore, sensorless force estimation plays an important role in the success of the force control process. Interaction force between the end effector and œxible objects is estimated using visual feedback instead of using force sensor [2]. By making a relationship between the end effector force and the deformation of the œxible object that can be visually detected. A comparison between the sensorless force control systems and force control system is performed in [1]. Where the reaction force is estimated by assuming that the internal force and other terms of the disturbance are identiGed precisely. Force is estimated and force error observer is designed in [3] using the velocity information without using the actual force sensor signal. Therefore, the associated strain gage problems are avoided, such as the narrow band width due to the natural frequency of the sensor. In this paper, actuator is used to estimate the externally applied forces or torques on a œxible multidegree-of-freedom system. Where the actuator $\mathrm{s}$ current and velocity are measured and disturbance is estimated. Surprisingly enough that disturbance which can be estimated from the actuator side contains two types of information. The Grst coupled information is related to the actuator s parameters such as the self varied-inertia torque and the actuator s torque ripple. The other coupled set of information is related to the

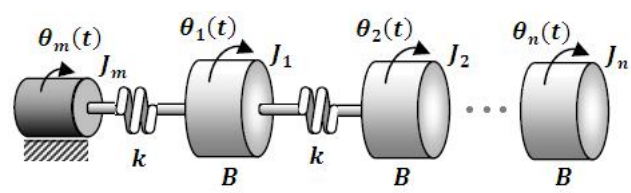

Fig. 1. Lumped œxible inertial system

plant, such as the reœected systems load and the externally applied torques or forces. Therefore, in this work the disturbance observer s structure is modicked in order to decouple these information out of the estimated total disturbance. In addition, the reœected load from the plant is proved to contain plant $\mathrm{s}$ parameters, dynamics and externally applied disturbances.

A sensorless force estimation algorithm is then introduced based on two actuator measurements (current and velocity), considering the reœected mechanical load that can be estimated by the actuator s parameters as a natural feedback from the plant [4].

This paper is organized as follows. In section 2, reœected mechanical waves are investigated and proved to contain enough information about the plant. Such as plant s parameters, dynamics and disturbances. In addition, disturbance observer is modiCed in order to decouple the reœected mechanical waves out of the total disturbance. In section 3, the sensorless force estimation algorithm is introduced. Section 4 includes the experimental results. Finally, remarks and conclusion are included in section 5 .

\section{Mechanical Waves Estimation}

\section{A. Mechanical Waves Analysis}

For a Multidegree-of-freedom œxible system with $n$ lumped masses connected to an actuator as shown in Fig.1. the equations of motion are [5]

$$
\begin{gathered}
J_{m} \ddot{\theta}_{m}+B\left(\dot{\theta}_{m}-\dot{\theta}_{1}\right)+k\left(\theta_{m}-\theta_{1}\right)=\tau_{m} \\
\vdots \\
J_{n} \ddot{\theta}_{n}-B\left(\dot{\theta}_{n-1}-\dot{\theta}_{n}\right)-k\left(\theta_{n-1}-\theta_{n}\right)=\tau_{\text {ext }}
\end{gathered}
$$

where, $J_{m}$ and $J_{i}$ are the actuator inertia and the inertia of the $i^{\text {th }}$ lumped mass. $k$ and $B$ are the uniform system s stiffness and damping coefCrients. $\theta_{m}$ and $\theta_{i}$ are the actuator angular 
position and the $i^{t h}$ lumped mass s position. $\tau_{m}$ and $\tau_{e x t_{i}}$ are actuator s torque and the external torque applied on the $i^{\text {th }}$ lumped mass, respectively. Putting the equations of motion together and solving for $B\left(\dot{\theta}_{m}-\dot{\theta}_{1}\right)+k\left(\theta_{m}-\theta_{1}\right)$ we obtain

$$
\begin{aligned}
\tau_{\text {ref }} & \triangleq \sum_{i=1}^{n} J_{i} \ddot{\theta}_{i}-\sum_{i=1}^{n} \tau_{\text {ext }} \\
& \triangleq B\left(\dot{\theta}_{m}-\dot{\theta}_{1}\right)+k\left(\theta_{m}-\theta_{1}\right)
\end{aligned}
$$

where $\tau_{\text {ref }}$ is the reœected torque wave from the dynamical system on the actuator. The previous equation shows that the reœected torque wave $\tau_{\text {ref }}$ carries enough information about the system s dynamics, parameters and external disturbances due to the system $\mathrm{s}$ interaction with the environment.

\section{B. Disturbance estimation}

Equation.1 represents the mechanical dynamics of the actuator without considering its parameters variation, that are given as follows [6]- [7]

$$
\begin{aligned}
J_{m} & =J_{m o}+\triangle J_{m} \\
k_{t} & =k_{t o}+\triangle k_{t}
\end{aligned}
$$

Where $k_{t}$ is the actuator s torque constant, $J_{m o}$ and $k_{t o}$ are the nominal actuator s inertia and torque constant while $\triangle J_{m}$ and $\triangle k_{t}$ are the variation between the actual and nominal actuator $\mathrm{s}$ inertia and torque constant. Rewriting eq. 1

$$
\left(J_{m o}+\triangle J_{m}\right) \ddot{\theta}_{m}=\left(k_{t o}+\triangle k_{t}\right) i_{m}-\tau_{r e f}
$$

re-arranging the terms

$$
J_{m o} \ddot{\theta}_{m}=k_{t o} i_{m}-\tau_{r e f}-\triangle J_{m} \ddot{\theta}_{m}+\triangle k_{t} i_{m}
$$

Where, $\triangle J_{m} \ddot{\theta}_{m}$ and $\triangle k_{t} i_{m}$ are the actuator s varied selfinertia torque and actuator $\mathrm{s}$ torque ripple. Therefore, the disturbance on the actuator side is

$$
d=-\tau_{r e f}-\underbrace{\triangle J_{m} \ddot{\theta}_{m}+\triangle k_{t} i_{m}}
$$

which indicates that the disturbance on the actuator side is composed of two components. The last two terms of the right hand side of eq. 6 represents the Grst disturbance component that is related to the actuator parameters s variations. While the second disturbance component $\tau_{\text {ref }}$ is due to the attached system with this actuator. Therefore, disturbance $d$ has to be estimated then reœected torque wave has to be decoupled out of it. From eq.5 the disturbance $d$ can be computed as follows

$$
d=J_{m o} \frac{d^{2} \theta_{m}}{d t^{2}}-k_{t o} i_{m}
$$

or estimated through a low pass with a corner frequency $g_{d i s t}$

$$
\widehat{d}=\frac{g_{d i s t}}{s+g_{\text {dist }}}\left[J_{m o} \ddot{\theta}_{m}-i_{m} k_{t o}\right]
$$

Therefore, the estimation error is

$$
\widetilde{d}=\widehat{d}-d
$$

introducing eq.7 and eq. 8 into eq. 9

$$
\widetilde{d}=\left[J_{m o} \ddot{\theta}_{m}-i_{m} k_{t o}\right] \frac{g_{d i s t}}{s+g_{d i s t}}-J_{m} \ddot{\theta}_{m}+i_{m} k_{t}
$$

multiplying eq.10 by $\left(s+g_{\text {dist }}\right)$ and making the following deGrition

$$
\xi \triangleq g_{d i s t} \triangle J \ddot{\theta}_{m}-s J_{m} \ddot{\theta}_{m}+g k_{t} i_{m}+s i_{m} k_{t}
$$

we obtain the following differential equation

$$
\frac{d}{d t} \widetilde{d}+g_{d i s t} \widetilde{d}=\xi
$$

which describes the estimation error dynamics and has the following solution

$$
\widetilde{d}(t)=e^{-g_{d i s t} t} \int_{o}^{t} e^{g_{d i s t} \tau} \xi(\tau) d \tau+c e^{-g_{d i s t} t}
$$

which indicates that the estimation error will exponentially decay, and the low pass corner frequency can be considered as the observer gain. In other words, changing the observer gain controls the speed of the estimation convergence.

$$
\begin{aligned}
t \longmapsto \infty & \longmapsto \widetilde{d} \longmapsto 0 \\
\tilde{d} \longmapsto 0 & \longmapsto \widehat{d} \longmapsto d
\end{aligned}
$$

The direct differentiation of the velocity signal can be avoided by using the following observer confguration to keep the noise ampliceation level as low as possible [8]- [9].

$$
\widehat{d}=\frac{g_{\text {dist }}}{s+g_{\text {dist }}}\left[J_{m o} \dot{\theta}_{m}+k_{t o} i_{m}\right]-g_{\text {dist }} J_{m o} \dot{\theta}_{m}
$$

\section{Reoected Torque Wave Decoupling}

Disturbance estimate obtained using eq.6, can be written as follows

$$
\widehat{\tau}_{r e f}=-\widehat{d}-\underbrace{\triangle J_{m} \ddot{\theta}_{m}+\triangle k_{t} i_{m}}
$$

which indicates that in order to decouple the reœcted wave out of the estimated disturbance, the self varied-inertia torque and actuator s torque ripple have to be determined Grst. Keeping in mind that variation between the actual and nominal actuator $\mathrm{s}$ parameters are inherent properties of the actuator. In other words, they are independent to the plant connected with the actuator. Therefore, they can be estimated from the unloaded actuator and in this case the reœected torque wave is eliminated from equations of motion eq. 1 .

$$
\begin{gathered}
\left(J_{m o}+\triangle J_{m}\right) \ddot{\theta}_{m}+B \dot{\theta}_{m}=\left(k_{t o}+\triangle k_{t}\right) i_{m} \\
J_{m o} \ddot{\theta}_{m}=k_{t o} i_{m}+d_{\text {par }}
\end{gathered}
$$

where $d_{\text {par }}$ is the Actuator s parameters variation disturbance

$$
d_{\text {par }}=\triangle k_{t} i_{m}-\triangle J_{m} \ddot{\theta}_{m}-B \dot{\theta}_{m}
$$

that can be estimated using the actuator s current and velocity through a low pass CHter as follows

$$
\widehat{d}_{p a r}=\frac{g_{d i s t}}{s+g_{d i s t}}\left[J_{m o} \dot{\theta}_{m}+i_{m} k_{t o}\right]-g_{d i s t} J_{m o} \dot{\theta}_{m}
$$

eq. 17 becomes

$$
\underline{\underline{d}}_{p a r}=-B \underline{\dot{\theta}}_{m}+\triangle k_{t o} \underline{i}_{m}-\triangle J_{m} \underline{\ddot{\theta}}_{m}
$$

where, $\widehat{\underline{d}}_{p a r}$ is the estimated parameters s disturbance vector data point, while $\underline{\dot{\theta}}_{m}, \underline{\ddot{\theta}}_{m}$ and $\underline{i}_{m}$ are data points vectors of 
actuator s velocity, acceleration and current. Putting eq.19 in the following matrix form

$$
\left[\begin{array}{lll}
\triangle k_{t} & -B & -\triangle J_{m}
\end{array}\right]\left[\begin{array}{c}
\underline{i}_{m} \\
\underline{\ddot{\theta}}_{m} \\
\underline{\underline{x}}_{m}
\end{array}\right]_{3 \times m}=\left[\widehat{d}_{\text {par }}\right]
$$

where $m$ is the number of data points of each vector.

$$
H \triangleq\left[\begin{array}{c}
\underline{i}_{m} \\
\underline{\ddot{\theta}}_{m} \\
\underline{-}_{m}
\end{array}\right]_{3 \times m}
$$

Matrix $H$ can be obtained from the actuator side by measuring it $\mathrm{s}$ current, velocity and acceleration. Indeed, obtaining the acceleration signal will result in high amplifeation of the noise level. Therefore, an appropriate differentiation techniques have to be used in this level [10].

Equation 20 represents an over-determined system, and it s solution has to minimize the norm square of errors.

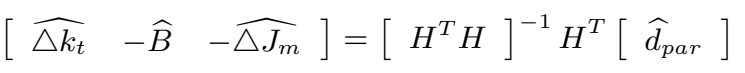

or

$$
\left[\begin{array}{lll}
\widehat{\triangle k_{t}} & -\widehat{B} & -\widehat{\triangle J_{m}}
\end{array}\right]=H^{\dagger}\left[{\widehat{d_{p a r}}}\right]
$$

where $H^{\dagger}$ is the pseudo-inverse of $H . \widehat{\triangle k_{t}}$ and $-\widehat{\triangle J_{m}}$ are the estimated actuator s torque ripple and varied self-inertia torque, respectively.

Rewriting eq.14 and replacing the actual parameter variations with the estimated ones, we get the estimated reœcted torque wave as follows

$$
\widehat{\tau}_{r e f}=\widehat{\triangle k_{t}} i_{m}-\widehat{\triangle J_{m}} \ddot{\theta}_{m}-\widehat{d}
$$

The direct differentiation of the velocity signal can be avoided by using the following observer s structure

$$
\begin{gathered}
\widehat{\tau}_{r e f}=G(s)\left[i_{m} \widehat{\triangle k_{t}}-\widehat{d}+g_{r e f} \widehat{\triangle J_{m}} \dot{\theta}_{m}\right]-g_{r e f} \widehat{\triangle J_{m}} \dot{\theta}_{m} \\
G(s)=\frac{g_{r e f}}{s+g_{r e f}}
\end{gathered}
$$

where $g_{\text {ref }}$ is reœcted torque observer s constant gain. The block diagram implementation of the reœected torque observer is shown in Fig.2.

\section{SENSORLESS TORQue ESTIMATION}

As the reoected torque wave is estimated, eq. 2 can be used to determine the external torques if the inertial mass $\mathrm{s}$ accelerations are available that requires taking measurement from each lumped mass of the œxible system. Instead, we propose an algorithm to estimate the œxible motion of each lumped mass of the œxible system. Then the estimated system $\mathrm{s}$ dynamics is used in eq. 2 to estimate the external torques.

\section{A. Uniform System s Parameters Estimation}

Since the system s stiffness and damping are inherent properties of the system. In other words, they are independent of the external applied torques. We assume that œxible system is free from external torques. Therefore, eq. 2 can be written as

$$
\widehat{\tau}_{r e f}=\sum_{i=1}^{n} J_{i} \ddot{\theta}_{i}=B\left(\dot{\theta}_{m}-\dot{\theta}_{1}\right)+k\left(\theta_{m}-\theta_{1}\right)
$$

This assumption is just made to determine the system parameters through an off-line experiment. Then, the estimated parameters along with the estimated dynamics will be used in order to estimate the external torque. However, determination of the system s uniform parameters $k$ and $B$ from eq.24 requires measuring the Crst mass $\mathrm{s}$ position. Surprisingly enough that if the œxible modes of the system

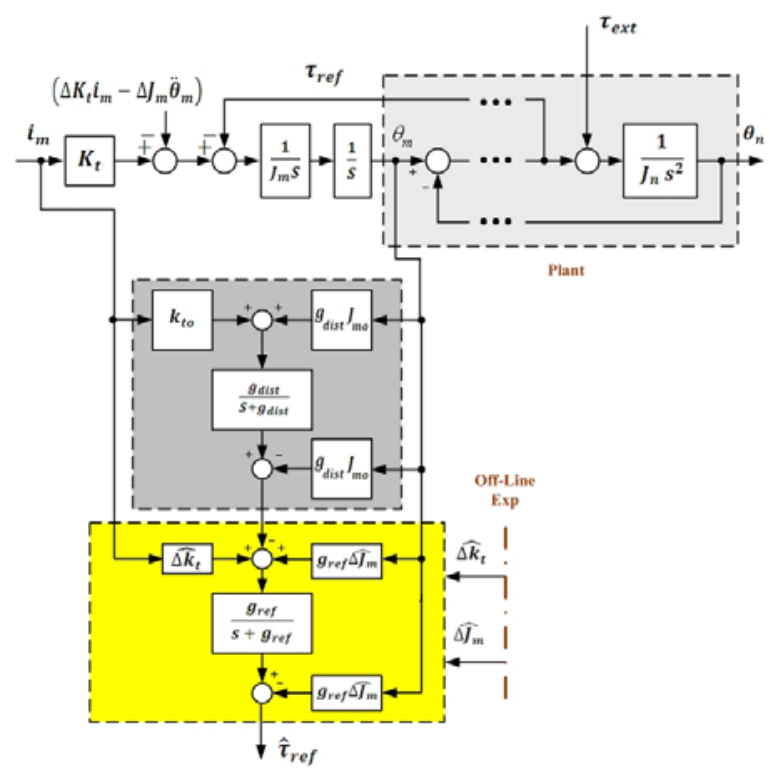

Fig. 2. Reœected torque observer s structure

are not excited, a single generalized-coordinate is enough to describe the motion of the œxible system that is no longer œxible instead of $n$ generalized-coordinate. Therefore, we can write

$$
\theta_{1}(t)=\theta_{2}(t)=\theta_{3}(t)=\ldots=\theta_{n}(t)=\theta(t)
$$

this equality is valid if and only if the control input is Cttered such that it contains zero energy at the system s resonance frequencies. Or Fourier synthesized to guarantee that its frequency content at the system s resonances is zero. Moreover, shaping the input by this way makes it possible to estimate the rigid motion of the œxible system by the following equation

$$
\widehat{\theta}(t)=\frac{1}{\sum_{i=1}^{n} J_{i}} \int_{o}^{t} \int_{o}^{t} \widehat{\tau}_{r e f} d \tau d \tau+c_{1} t+c_{2}
$$

where $\widehat{\theta}(t)$ is the rigid motion position estimate. And eq.26 is only valid through a narrow region of the œxible system $\mathrm{s}$ frequency range. Therefore, within this frequency range the parameters estimation process has to be performed.

Rewriting eq.24 and using $\widehat{\theta}(t)$ instead of $\theta_{1}(t)$

$$
\widehat{\tau}_{r e f}=B\left(\dot{\theta}_{m}-\widehat{\dot{\theta}}\right)+k\left(\theta_{m}-\widehat{\theta}\right)
$$

and deCfning the velocity and position differences as follows

$$
\begin{aligned}
& \underline{\xi} \triangleq\left(\theta_{m}-\widehat{\theta}\right) \\
& \underline{\eta} \triangleq\left(\dot{\theta}_{m}-\widehat{\dot{\theta}}\right)
\end{aligned}
$$

where $\underline{\xi}$ and $\underline{\eta}$ are vectors of data points, Similarly, $\widehat{\underline{\tau}}_{r e f}$ is the estimated reœected torque data point vector, rewriting eq.27 in the following matrix form

$$
\begin{gathered}
{\left[\begin{array}{ll}
\underline{\xi} & \underline{\eta}
\end{array}\right]_{n \times 2}\left[\begin{array}{c}
k \\
B
\end{array}\right]_{2 \times 1}=\left[\begin{array}{l}
\widehat{\underline{\tau}}_{r e f}
\end{array}\right]_{n \times 1}} \\
G \triangleq\left[\begin{array}{ll}
\underline{\xi} & \underline{\eta}
\end{array}\right]
\end{gathered}
$$

solving eq. 28 for the system parameters vector we obtain

$$
\left[\begin{array}{l}
\widehat{k} \\
\widehat{B}
\end{array}\right]=\left[G^{T} G\right]^{-1} G^{T}\left[\widehat{\underline{\tau}}_{r e f}\right]
$$




$$
\left[\begin{array}{l}
\widehat{k} \\
\widehat{B}
\end{array}\right]=G^{\dagger}\left[\widehat{\underline{\underline{\tau}}}_{r e f}\right]
$$

Where $G^{\dagger}$ is the pseudo inverse of $G, \widehat{k}$ and $\widehat{B}$ are the estimates of the system s uniform stiffness and damping coefGeients. The previous procedure is considered as an off-line parameters estimation experiment that is performed in a certain system s frequency range to estimate œxible system s uniform stiffness and damping.

\section{B. Flexible Motion Estimation}

Equation 26 is valid in narrow region of the system s frequency range. In addition, it estimates the motion of the œexible system when its rigidly behaving. Therefore, eq.26 is not enough to estimate the œxible system s motion, where the amplitude ratios between the masses are no longer unity and masses are no longer in phase.

Recalling eq. 2 and replacing the actual parameters with the estimated ones

$$
\widehat{\tau}_{r e f}=\widehat{B}\left(\dot{\theta}_{m}-\dot{\theta}_{1}\right)+\widehat{k}\left(\theta_{m}-\theta_{1}\right)
$$

re-arranging the terms

$$
\widehat{B} \dot{\theta}_{1}+\widehat{k} \theta_{1}=\widehat{B} \dot{\theta}_{o}+\widehat{k} \theta_{o}-\widehat{\tau}_{r e f}
$$

solving the Grst order differential equation for $\theta_{1}(t)$ that has to be denoted as $\widehat{\theta}_{1}(t)$ since it depends on observed variable $\widehat{\tau}_{r e f}$ and estimated parameters such as $\widehat{k}$ and $\widehat{B}$.

$$
\widehat{\theta}_{1}(t)=e^{-\frac{\widehat{B}}{k} t} \int_{o}^{t} \beta e^{\widehat{\widehat{B}}} \tau
$$

where

$$
\begin{gathered}
\beta \triangleq \frac{\alpha}{\widehat{B}} \\
\alpha \triangleq \widehat{B} \dot{\theta}_{o}+\widehat{k} \theta_{o}-\widehat{\tau}_{r e f}
\end{gathered}
$$

$\widehat{\theta}_{1}(t)$ is the position estimate of the Crst mass and eq.33 is valid through the entire system $s$ frequency range regardless to the frequency content of the forcing function.

Recalling the Grst œxible system s equation of motion and replacing the Grst mass position with its estimate we obtain

$$
\widehat{B} \dot{\theta}_{2}+\widehat{k} \theta_{2}=J_{1} \widehat{\ddot{\theta}}_{1}-\widehat{B}\left(\dot{\theta}_{o}-\widehat{\dot{\theta}}_{1}\right)-\widehat{k}\left(\theta_{o}-\theta_{1}\right)+\widehat{B} \widehat{\dot{\theta}}_{1}+\widehat{k} \widehat{\theta}_{1}
$$

solving for $\widehat{\theta}_{2}(t)$ we obtain

$$
\widehat{\theta}_{2}(t)=e^{-\frac{\widehat{k}}{\widehat{B}} t} \int_{o}^{t} \zeta e^{\widehat{\widehat{k}} \widehat{B}} \tau t \tau+e^{-\frac{\widehat{B}}{k} t} c_{2}
$$

where

$$
\begin{gathered}
\zeta \triangleq \frac{\gamma}{\widehat{B}} \\
\gamma \triangleq J_{1} \widehat{\ddot{\theta}}_{1}-\widehat{B}\left(\dot{\theta}_{o}-\widehat{\dot{\theta}}_{1}\right)-\widehat{k}\left(\theta_{o}-\theta_{1}\right)+\widehat{B} \widehat{\dot{\theta}}_{1}+\widehat{k} \widehat{\theta}_{1}
\end{gathered}
$$

In general, the position estimate of the $i^{\text {th }}$ lumped mass is

$$
\widehat{\theta}_{i}(t)=e^{-\frac{\widehat{k}}{\widehat{B}} t} \int_{o}^{t} \Omega e^{\frac{\widehat{k}}{\widehat{B}} \tau} d \tau+e^{-\frac{\widehat{B}}{k} t} c_{i}
$$

where

$$
\begin{gathered}
\Omega \triangleq \frac{\Psi}{\widehat{B}} \\
\Psi \triangleq g\left(J_{i-1}, \widehat{\theta}_{i-1}, \widehat{\dot{\theta}}_{i-1}, \widehat{\ddot{\theta}}_{i-1}, \widehat{k}, \widehat{B}\right)
\end{gathered}
$$

eq.36 can be considered as a set of position observers that are recursively estimating positions of system s lumped masses. Surprisingly enough that if a proper differentiation tool is used, the velocity and the accelerations of each mass can be obtained without taking any single measurement from the œxible system s side.

\section{External Torque Estimation}

Since the oxible system s dynamics can be observed and the uniform parameters can be estimated, eq. 2 can be used in order to estimate the external torques or disturbance due to the system $\mathrm{s}$ interaction with the environment.

$$
\widehat{\tau}_{e x t}=\sum_{i=1}^{n} J_{i} \widehat{\ddot{\theta}}_{i}-\widehat{\tau_{r e f}}
$$

Where $\widehat{\tau}_{\text {ext }}$ is the estimate of the external applied forces on

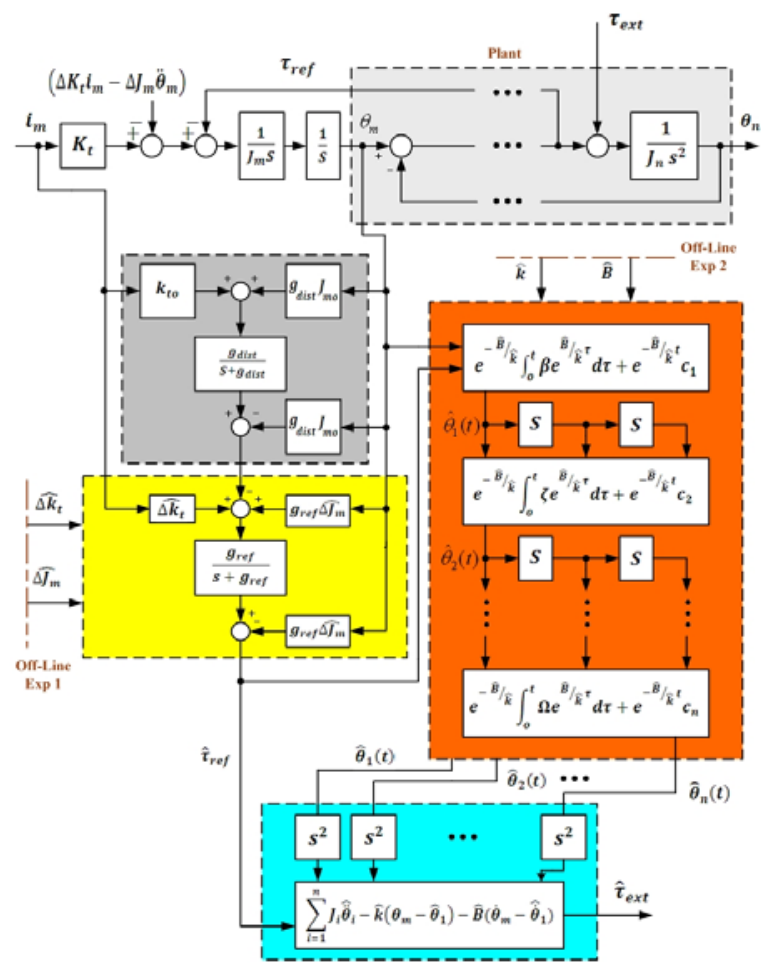

Fig. 3. External applied torque estimation

the plant. The sensorless torque estimation process is illustrated in Fig.3. Indeed, the word Sensorless is not precise, since one must sense or measure some variable to obtain some information as a basis of estimating the unknown variables. In this context the word sensorless refers to the œxible plant that is kept free from any measurement and only two measurement are required from the actuators side as shown in Fig.3. Where actuator s current and velocity are measured and used to estimate the total disturbance, then the reœected torque wave is decoupled out of this disturbance and used as an input for a chain of œxible motion observers that provide eq. 37 with the necessary entries to estimate the external torque. The previous procedure requires performing a couple of offline experiments based on the same actuators parameters current and velocity. The Grst experiment is performed to estimate the parameters variation disturbance in order to decouple the reœcted torque wave, while the second experiment is performed in order to estimate the uniform system s parameters.

\section{EXPERIMENTAL RESULTS}

The experimental setup consists of a multidegree-of-freedom inertial œxible system attached to an actuator that is used as a platform for measurements and estimations. Optical encoders are attached to each mass of the system in order to verify the performance of the positions observers. Table.I summarizes the parameters used in the following experiments 


\begin{tabular}{|c|c|c|c|}
\hline Parameter & Value & Parameter & Value \\
\hline$J_{1}$ & $5152.9 \mathrm{gcm}^{2}$ & $J_{3}$ & $6192.7 \mathrm{gcm}^{2}$ \\
$J_{2}$ & $5152.9 \mathrm{gcm}^{2}$ & $f_{1}$ & $1 \mathrm{rad} / \mathrm{sec}^{2}$ \\
$f_{2}$ & $2 \mathrm{rad} / \mathrm{sec}^{2}$ & $f_{3}$ & $3 \mathrm{rad} / \mathrm{sec}$ \\
$f_{4}$ & $4 \mathrm{rad} / \mathrm{sec}$ & $k_{t o}$ & $40.6 \mathrm{mNm} / \mathrm{A}$ \\
$k_{b}$ & $235 \mathrm{rpm} / \mathrm{v}$ & $J_{m o}$ & $209 \mathrm{gcm}$ \\
$g_{\text {dist }}$ & $100 \mathrm{rad} / \mathrm{sec}$ & $g_{l p f}$ & $100 \mathrm{rad} / \mathrm{sec}$ \\
\hline
\end{tabular}

\begin{tabular}{cccccc} 
Par & 1st Exp & 2nd & 3rd & 4th & 5th \\
\hline$\widehat{k} \mathrm{KN} / \mathrm{m}$ & 1.579 & 1.533 & 1.645 & 1.511 & 1.562 \\
$\widehat{B} \mathrm{Nsec} / \mathrm{m}$ & 0.088 & 0.087 & 0.088 & 0.089 & 0.089 \\
\hline
\end{tabular}

\section{A. Rigid Body Motion Estimation Experiment}

This experiment is performed at the system s low frequency range that is achieved by Htering the forcing function to avoid exciting the system s œxible modes. Fig. 4 shows the response of a 3 Dof œxible system, where all the amplitude ratios are unity and the masses are in phase. The results shown in Fig.4 indicates the validity of eq.26 in this frequency range. Where the estimated rigid body position is following the actual position of the rigid system. The frequency of

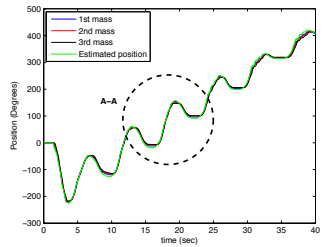

(a) $f_{1}=1 \mathrm{rad} / \mathrm{sec}$

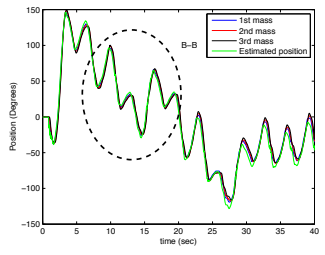

(c) $f_{2}=2 \mathrm{rad} / \mathrm{sec}$

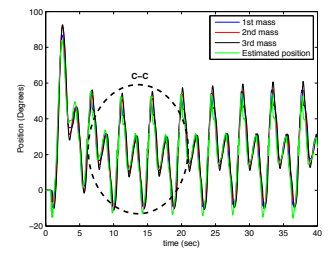

(e) $f_{3}=3 \mathrm{rad} / \mathrm{sec}$

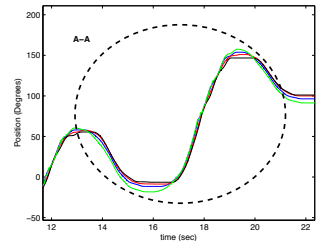

(b) Mag plot of a

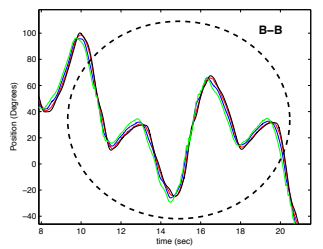

(d) Mag plot of c

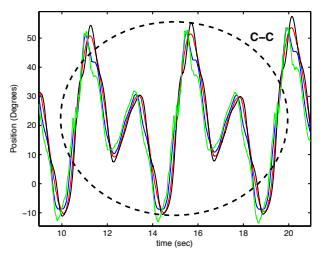

(f) Mag plot of a

Fig. 4. Rigid body motion estimation

the forcing function is increased gradually in order to determine the frequency region at which eq. 26 is valid. It turns out that eq.26 is valid below $3 \mathrm{rad} / \mathrm{sec}$. Therefore, parameters estimation experiment has to be performed in this frequency range for this particular system as its based on eq. 26 .

\section{B. Parameters Estimation Experiment}

Equation 30 is used to estimate the œxible system s stiffness and damping. Therefore, estimate of the reœected torque wave is computed along with the data matrix $G$, which depends on the actuator $\mathrm{s}$ and rigid system s position. Table.II shows the experimental system s parameters obtained by Eq.30. In order to obatain more reliable results the experiment was repeated $l$ times and the obtained

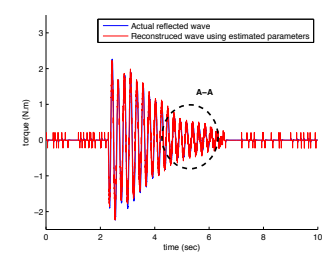

(a) reœected and reconstructed reœeted torque wave

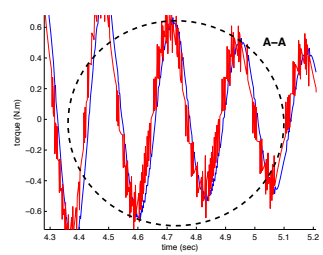

(b) Magnifed plot of a
Fig. 5. Parameters estimation experiment

average values are

$$
\begin{aligned}
& \widehat{k}_{a v g}=\frac{\sum_{i=1}^{l} k_{i}}{n}=\frac{30.9306}{20}=1.54653 \mathrm{kN} / \mathrm{m} \\
& \widehat{B}_{a v g}=\frac{\sum_{i=1}^{l} B_{i}}{n}=\frac{1.6866}{20}=0.08433 \mathrm{Nsec} / \mathrm{m}
\end{aligned}
$$

The difference between these parameters and the actual ones that are known before hand is less than 5 percent, that can be acceptable for certain applications. Fig. 5 shows both the original reœected wave and the reconstructed one using the estimated parameters using Eq.27.

\section{Flexible Motion Estimation Experiment}
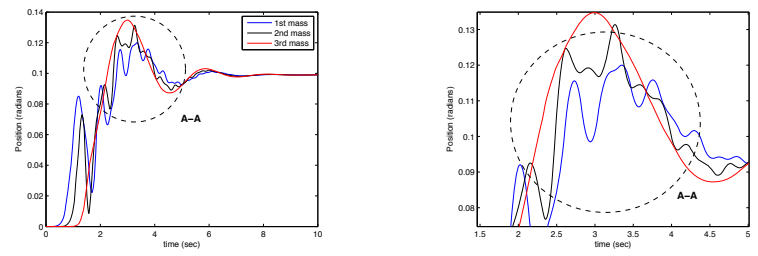

Fig. 6. Flexible Oscillation of a 3DOF Dynamical System

Using the recursive œxible motion observers eq.36, œxible motion can be estimated regardless to the frequency content of the forcing function. Fig.6 shows the response of the 3 Dof axible system to an arbitrary forcing function that forces the system to œxibly oscillate. The position estimates of each lumped mass of the system is shown in Fig.7 and compared with the actual optical encoder measurement. The results show the validity of the proposed algorithm to estimate the motion of the multidegree-of-freedom œxible system.

\section{Torque Estimation Experiment}

Estimating the external torque applied on the œxible system using eq.37 requires estimating the œxible system s dynamics and the estimate of the reœected torque wave ${ }^{1}$. Experimentally a sinusoidal torque disturbance is added to the œxible system and simply measured and compared with the estimated one. The frequency of the externally applied sinusoidal torque was varied between 1-5 rad/sec. Fig.8-a

\footnotetext{
${ }^{1}$ Experimentally reœected torque wave is assumed to be equal to the disturbance estimated from the actuator, that cannot be generalized since the parameters variation disturbance can effect the results
} 


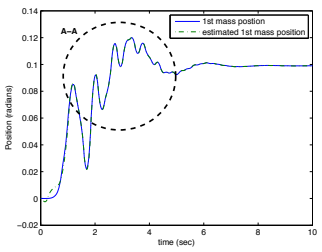

(a) $1^{\text {st }}$ Mass and Its Estimate

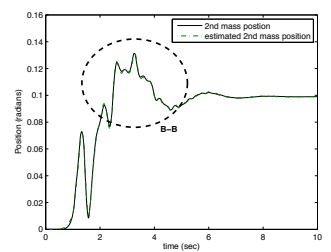

(c) $3^{\text {rd }}$ Mass and Its Estimate

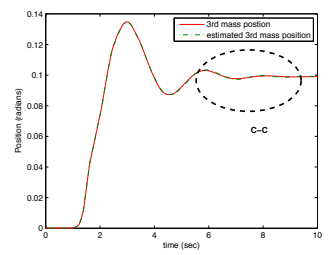

(e) Magni(fed Plot B-B

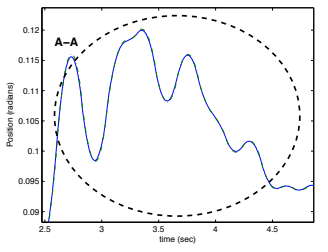

(b) $2^{\text {nd }}$ Mass and Its Estimate

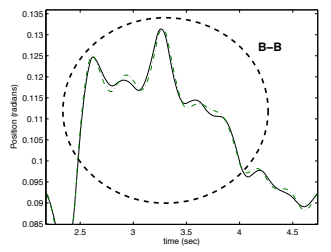

(d) Magni(fed Plot A-A

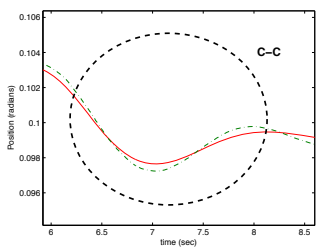

(f) MagniGed Plot C-C
Fig. 7. Flexible body motion estimation experimental results

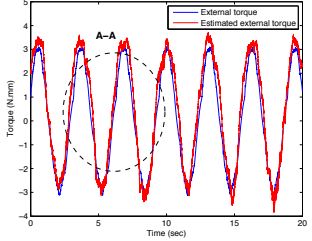

(a) Estimated and external torque- $\left.\tau_{\text {ext }}\right|_{f=2 \mathrm{rad} / \mathrm{sec}}$

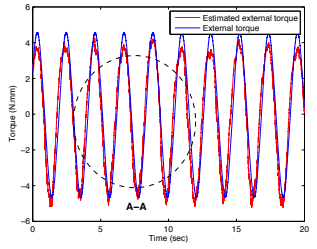

(c) Estimated and external torque- $\left.\tau_{\text {ext }}\right|_{f=4 \mathrm{rad} / \mathrm{sec}}$

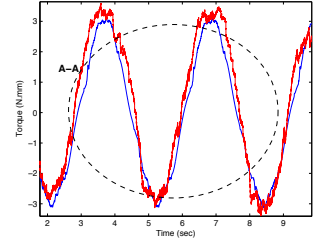

(b) Magniđed plot of A-A

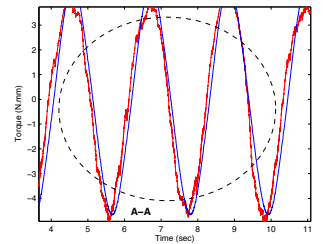

(d) Magni(אed Plot A-A
Fig. 8. External torque estimation

shows the estimated and actual torque of $2 \mathrm{rad} / \mathrm{sec}$ frequency, while Fig.8-c shows the same result when the external torque s frequency is $4 \mathrm{rad} / \mathrm{sec}$.

\section{CONCLUSion}

This paper introduces an algorithm for estimating the externally applied disturbance due to œxible system $\mathrm{s}$ interaction with the environment. The proposed algorithm is treating the disturbances and system s œxibility differently. Disturbance is not only used as a step toward the accomplishment of robust motion control system but treated as coupled signal that contains information about sys- tem s parameters, dynamics and external forces/torques. In addition, œxibility is treated as an effeient tool by shaping the control input to obtain certain behavior of the Multidegree-of-freedom system. In other words, making it possible to minimize number of generalizedcoordinates describing œexible system s motion, that in turn makes it possible to determine system parameters easier as too many unknowns are dropped.

The reœected mechanical waves are decoupled out of the total disturbance and proved to contain enough information about the dynamical system. Then, system is rigidly excited with a pre-CHtered control input and rigid motion is estimated. Moreover, rigid system $\mathrm{s}$ position is used along with reœcted torque wave estimate to estimate the uniform system $\mathrm{s}$ stiffness and damping with less than 5 percent error when compared with the actual parameters that are known before hand. Then, the estimated parameters are used along with the reœected mechanical waves and actuator parameters to design a chain of œxible motion observers that are recursively estimating œxible motion of each lumped mass of the system. Proper differentiation of the position estimates makes all the system s dynamics available. Eventually, the estimated dynamics along with the reœcted torque waves are used to estimate the externally applied torque. Experimental results show the validity of the algorithm that can be used in order to accomplish a sensorless force control assignments.

\section{ACKNOWLEDGMENT}

The authors gratefully acknowledge the Crnancial assistance provided by SanTez project number 00183.STZ.2007-2.

\section{REFERENCES}

[1] A. Kato and K. Ohnishi, Robust force sensorless control in motion control system, $f$ in $A M C 06 f$. Istanbul, Turkey: IEEE, 2006, pp. $165^{-}$ 170.

[2] H. Huang, D. Sun, J. K. Mills, and W. J. Li, Visual-based impedence force control of three-dimentional cell injection system, $f$ in Proc. IEEE International Conference on Robotics and Automation 07, Roma, Italy, Apr. 2007, pp. 4196 4201.

[3] W. Iida and K. Ohnishi, Sensorless force control with force error observer, $f$ in ICIT 03f. Maribor, Slovenia: IEEE, 2003, pp. 157 162.

[4] W.J.O Connar, Wave-based analysis and control of lump-modeled œxible robots, $f$ in Proc. IEEE Transaction on robotics 07, vol. 23, Apr. 2007, pp. $342 \smile 352$.

[5] I. S. M. Khalil and A. Sabanovic, Sensorless wave based control of œxible structures using actuator as a signle platform for estimation and control,f IREACO, vol. 2, no. 1, pp. 83`90, Jan 2009.

[6] K. Ohnishi, M. Shibata, and T. Murakami, Motion control for advanced mechatronics, $f$ in Proc. IEEE/ASME Transaction On Mechatronics 96, vol. 1, Mar. 1996, pp. 56 67.

[7] T. Murakami and K. Ohnishi, Robust and adaptive control strategies, $f$ in Proc. IFAC International Control for Intelligent Automation 92, vol. 1, Italy, Oct. 1992, pp. $367^{\sim} 372$.

[8] , Observer-based motion control-application to robust control and parameters identifcation, $f$ in Proc. IEEE 93, vol. 1, Jun. 1993, pp. 1`6.

[9] T. Murakami, F. Yu, and K. Ohnishi, Torque sensorless control in multidegreeo-of-freedom manipulator, $f$ in Proc. IEEE Transaction On Industrial Electronics 07, vol. 40, Apr. 1993, pp. 259־ 265.

[10] T. Tsuji, M. Mizuochi, H. Nishi, and K. Ohnisi, A velocity measurement method for acceleration control,f in Proc. IEEE 05, vol. 1, Mar. 2005, pp. 19431947. 